\title{
Canadian Thoracic Society recommendations for management of chronic obstructive pulmonary disease - 2008 update - highlights for primary care
}

\author{
Denis $\mathrm{E}^{\prime}$ Donnell $\mathrm{MD}^{1 *+}$, Paul Hernandez $\mathrm{MD}^{2 * \neq}$, Alan Kaplan $\mathrm{MD}^{3}$, Shawn Aaron $\mathrm{MD}^{4 *}$, \\ Jean Bourbeau $M D^{5 *}$, Darcy Marciniuk $M D^{6 *}$, Meyer Balter $\mathrm{MD}^{7}$, Gordon Ford $\mathrm{MD}^{8}$, \\ Andre Gervais $M D^{9}$, Yves Lacasse $M D^{10}$, Francois Maltais $M D^{10}$, Jeremy Road $M D^{11}$, \\ Graeme Rocker $M D^{2}$, Don Sin $M D^{11}$, Tasmin Sinuff $M D^{12}$, Nha Voduc $M D^{4}$
}

DE O'Donnell, P Hernandez, A Kaplan, et al. Canadian Thoracic Society recommendations for management of chronic obstructive pulmonary disease - 2008 update - highlights for primary care. Can Respir J 2008;15(Suppl A):1A-8A.

Chronic obstructive pulmonary disease (COPD) is a major respiratory illness in Canada that is preventable and treatable but unfortunately remains underdiagnosed. The purpose of the present article from the Canadian Thoracic Society is to provide up-to-date information so that patients with this condition receive optimal care that is firmly based on scientific evidence. Important summary messages for clinicians are derived from the more detailed Update publication and are highlighted throughout the document. Three key messages contained in the update are: use targeted screening spirometry to establish a diagnosis and initiate prompt management (including smoking cessation) of mild COPD; improve dyspnea and activity limitation in stable COPD using new evidence-based treatment algorithms; and understand the importance of preventing and managing acute exacerbations, particularly in moderate to severe disease.

Key Words: Chronic obstructive pulmonary disease; Management; National guidelines
Recommandations de la Société canadienne de thoracologie pour prendre en charge la maladie pulmonaire obstructive chronique - Mise à jour 2008 : Faits saillants des soins primaires

La maladie pulmonaire obstructive chronique (MPOC) est une grave maladie respiratoire au Canada. Elle peut être prévenue et traitée, mais demeure malheureusement sous-diagnostiquée. Le présent article de la Société canadienne de thoracologie vise à fournir de l'information à jour pour que les patients atteints de cette maladie reçoivent des soins optimaux solidement étayés par des preuves scientifiques. Des messages résumés importants à l'intention des cliniciens sont tirés de la mise à jour plus détaillée et soulignés tout au long du document. La mise à jour contient trois messages clés : utiliser la spirométrie de dépistage ciblée pour diagnostiquer et prendre rapidement en charge la MPOC bénigne (y compris le renoncement au tabac), réduire la dyspnée et les contraintes aux activités en cas de MPOC stable au moyen de nouveaux algorithmes de traitement probants et comprendre l'importance de la prévention et de la prise en charge des exacerbations aiguës, notamment en présence d'une maladie modérée à grave.

${ }^{1}$ Queen's University, Kingston, Ontario; ${ }^{2}$ Dalhousie University, Halifax, Nova Scotia; ${ }^{3}$ Family Physicians Airway Group of Canada, Richmond

Hill; ${ }^{4}$ University of Ottawa, Ottawa, Ontario; ${ }^{5} \mathrm{McGill}$ University, Montreal, Quebec; ${ }^{6}$ University of Saskatchewan, Saskatoon,

Saskatchewan; ${ }^{7}$ University of Toronto, Toronto, Ontario; ${ }^{8}$ University of Calgary, Calgary, Alberta; ${ }^{9}$ University of Montreal, Montreal;

${ }^{10}$ University of Laval, Sainte-Foy, Quebec; ${ }^{11}$ University of British Columbia, Vancouver, British Columbia; ${ }^{12} \mathrm{McM}$ aster University,

Hamilton, Ontario

* Member of the editorial committee; ${ }^{\dagger}$ Chair, Canadian Thoracic Society COPD Guidelines Development Committee; ${ }^{\ddagger}$ Chair, Canadian Thoracic Society COPD Guidelines Dissemination and Implementation Committee

Correspondence: Dr Denis E O'Donnell, Division of Respiratory and Critical Care Medicine, Department of Medicine, Queen's University,

102 Stuart Street, Kingston, Ontario K7L 2V6. Telephone 613-548-2339, fax 613-549-1459, e-mail odonnell@queensu.ca 


\section{DEFINITION AND PATHOPHYSIOLOGY}

Chronic obstructive pulmonary disease (COPD) is a respiratory disorder largely caused by smoking, and is characterized by progressive, partially reversible airway obstruction and lung hyperinflation, systemic manifestations, and increasing frequency and severity of exacerbations.

COPD is characterized by persistent inflammation of airways, lung parenchyma and its vasculature. The inflammatory process in COPD is different from that in asthma. Expiratory flow limitation is the pathophysiological hallmark of COPD. Expiratory flow limitation with dynamic collapse of the small airways compromises the ability of patients to expel air during expiration, resulting in air trapping and lung hyperinflation. Acute-on-chronic hyperinflation has been shown to contribute to shortness of breath during exercise and acute exacerbation in COPD.

COPD is associated with multiple comorbid conditions. These include ischemic heart disease, osteopenia and osteoporosis, glaucoma and cataracts, cachexia and malnutrition, anemia, peripheral muscle dysfunction, cancer and the metabolic syndrome. Rates of recognized anxiety and depression in COPD vary from $20 \%$ to $50 \%$ and increase with disease severity.

\section{EPIDEMIOLOGY IN CANADA}

COPD is underdiagnosed. COPD is a common respiratory illness in Canada affecting at least 700,000 adults and is now the fourth leading cause of death.

Current prevalence statistics likely underestimate the true prevalence of COPD in Canada. Approximately 4.4\% of Canadians aged 35 years or older (more than 700,000 adults) have COPD based on self-reporting of diagnoses made by health care professionals. The prevalence of COPD increases with increasing age. Women have a higher prevalence of COPD than men in all age groups except for the 75 years and older group. In 2003, 5366 men and 4503 women died of COPD, making COPD the fourth leading cause of death in Canada. Mortality rates have increased over the past 15 years, particularly in women.

\section{CLINICAL ASSESSMENT}

Most individuals with COPD are not diagnosed until the disease is well advanced. Targeted testing of symptomatic individuals with risk factors for the development of COPD followed by intensive smoking cessation counselling can change the progression of disease.

Mass screening of asymptomatic individuals for COPD is not supported by the current evidence and therefore is not recommended. The following criteria are recommended to help the family physician decide who to target for spirometry in their practice so as to establish early diagnosis in individuals at risk of COPD.

Patients who are older than 40 years of age and who are current or ex-smokers should undertake spirometry if they answer yes to any one of the following questions:

1 . Do you cough regularly?

2. Do you cough up phlegm regularly?
3. Do even simple chores make you short of breath?

4. Do you wheeze when you exert yourself, or at night?

5. Do you get frequent colds that persist longer than those of other people you know?

Episodes of acute bronchitis in a smoker may represent the first clinical presentation of COPD - spirometry should be obtained after the acute episode has resolved and the patient is clinically stable.

Tobacco consumption should be quantified:

$$
\begin{gathered}
\text { total pack years }= \\
\text { (number of cigarettes smoked per day } \div 20) \times \text { number } \\
\text { of years of smoking. }
\end{gathered}
$$

Individual susceptibility for the development of COPD varies, so no minimum number of pack years is required to place an individual at risk.

COPD management decisions should be made on an individual basis and should not be based exclusively on spirometry results but also on an assessment of severity of dyspnea and disability, which are assessed by using the Medical Research Council dyspnea scale (Tables 1 and 2).

Clinical assessment begins with a thorough history and physical examination. Occupational or environmental exposures to cigarette smoke and other lung irritants should be recorded. Symptoms and signs related to the COPD, complications (eg, leg edema in cor pulmonale) and comorbidities should be identified. Inquiry concerning frequency and severity of exacerbations is crucial to management decisions. Current medical treatment should be reviewed. Physical examination, although important, is not usually diagnostic except in the presence of very severe airflow limitation.

Postbronchodilator spirometry is required to evaluate the presence and severity of airway obstruction (Table 2). Additional pulmonary function and exercise tests may be undertaken in selected patients for a more complete clinical characterization of the COPD phenotype. Arterial blood gas measurements should be considered in patients with forced expiratory volume in $1 \mathrm{~s}\left(\mathrm{FEV}_{1}\right)$ of less than $40 \%$ predicted (with a resting arterial oxygen saturation of less than $90 \%$ ) to assess for evidence of hypoxemia or hypercapnia. Chest $\mathrm{x}$-rays and other investigations are not diagnostic of COPD but are often required to assess comorbidities.

COPD and asthma are fundamentally different.

In a small proportion of patients, diagnostic differentiation can be challenging and may require additional investigation or specialist referral. Clinical differences between asthma and COPD can usually help in making a correct diagnosis (Table 3). Patients with a large improvement in $\mathrm{FEV}_{1}$ (eg, greater than $0.4 \mathrm{~L}$ ) acutely following inhaled short-acting bronchodilators or following treatment with inhaled or oral steroids likely have an asthmatic component. 
TABLE 1

The Medical Research Council dyspnea scale

\begin{tabular}{ll}
\hline Grade & Description \\
\hline 1 & $\begin{array}{l}\text { Not troubled by breathlessness except with strenuous } \\
\text { exercise }\end{array}$ \\
2 & $\begin{array}{l}\text { Troubled by shortness of breath when hurrying on the level or } \\
\text { walking up a slight hill }\end{array}$ \\
3 & Walks slower than people of the same age on the level because of \\
breathlessness or has to stop for breath when walking at own \\
pace on the level \\
Stops for breath after walking about 100 yards ( 90 m) \\
or after a few minutes on the level \\
Too breathless to leave the house or breathless when dressing \\
or undressing
\end{tabular}

Other conditions included in the differential diagnosis of older patients presenting with progressive dyspnea include cardiovascular conditions, pulmonary vascular disease, severe deconditioning, obesity, anemia, interstitial lung disease and neuromuscular disease.

\section{MANAGEMENT}

COPD is treatable at any stage of the illness. A management strategy consisting of combined pharmacotherapy and nonpharmacotherapeutic interventions can effectively improve symptoms, activity levels and quality of life at all levels of disease severity.

The goals of COPD management include prevention of disease progression; reduction of the frequency and severity of exacerbations; alleviation of dyspnea and other respiratory symptoms; improvement of exercise tolerance; prompt treatment of exacerbations and complications of the disease; improvement of health status; and reduction of mortality. A stepwise, comprehensive management approach is required to achieve these goals (Figure 1).

\section{Education}

Education of the patient and family with supervision and support based on disease-specific self-management principles is valuable, and should be part of the continuum of optimal COPD management in Canada.

COPD education needs to be individualized and will vary with disease severity. Specific educational interventions, such as self-management programs and smoking cessation counselling, have been shown to reduce health resource utilization related to management of acute exacerbations. Components of COPD education include effective inhaler technique, early recognition and treatment of acute exacerbations, identification of community resources and end-of-life care issues.

\section{Smoking cessation}

COPD is preventable. Smoking cessation is the single most effective intervention to reduce the risk of developing COPD and the only intervention that has been shown to slow the rate of lung function decline.
TABLE 2

Canadian Thoracic Society chronic obstructive pulmonary disease (COPD) classification of severity by symptoms and disability*, and impairment of lung function

\begin{tabular}{ll}
\hline Classification by symptoms and disability \\
\hline COPD stage & Symptoms \\
\hline Mild & $\begin{array}{c}\text { Shortness of breath from COPD } \\
\text { or walking up a slight hill (MRC } 2)\end{array}$
\end{tabular}

Moderate Shortness of breath from $\mathrm{COPD}^{\dagger}$ causing the patient to stop after walking approximately $100 \mathrm{~m}$ (or after a few minutes) on the level (MRC 3 to 4 )

Severe $\quad$ Shortness of breath from COPD ${ }^{\dagger}$ resulting in the patient being too breathless to leave the house, breathless when dressing or undressing (MRC 5), or the presence of chronic respiratory failure or clinical signs of right heart failure

Classification by impairment of lung function

\begin{tabular}{ll}
\hline COPD stage & Spirometry (postbronchodilator) \\
\hline Mild & $\mathrm{FEV}_{1} \geq 80 \%$ predicted, $\mathrm{FEV}_{1} / \mathrm{FVC}<0.7$ \\
Moderate & $50 \% \leq \mathrm{FEV}_{1}<80 \%$ predicted, $\mathrm{FEV}_{1} / \mathrm{FVC}<0.7$ \\
Severe & $30 \% \leq \mathrm{FEV}_{1}<50 \%$ predicted, $\mathrm{FEV}_{1} / \mathrm{FVC}<0.7$ \\
Very severe & $\mathrm{FEV}_{1}<30 \%$ predicted, $\mathrm{FEV}_{1} / \mathrm{FVC}<0.7$ \\
\hline
\end{tabular}

${ }^{*}$ Postbronchodilator forced expiratory volume in $1 s\left(F E V_{1}\right)$ to forced vital capacity (FVC) ratio less than 0.7 is required for the diagnosis of COPD to be established; $t$ In the presence of non-COPD conditions that may cause shortness of breath (eg, cardiac dysfunction, anemia, muscle weakness, metabolic disorders), symptoms may not appropriately reflect COPD disease severity. Classification of COPD severity should be undertaken with care in patients with comorbid diseases or other possible contributors to shortness of breath. MRC Medical Research Council dyspnea scale

TABLE 3

Clinical differences between asthma and chronic obstructive pulmonary disease (COPD)

\begin{tabular}{lll}
\hline & \multicolumn{1}{c}{ Asthma } & \multicolumn{1}{c}{ COPD } \\
\hline Age of onset & Usually $<40$ years & Usually $>40$ years \\
Smoking history & Not causal & Usually $>10$ pack-years \\
Sputum production & Infrequent & Often \\
Allergies & Often & Infrequent \\
Disease course & Stable (with & Progressive worsening \\
& exacerbations) & (with exacerbations) \\
Spirometry & Often normalizes & May improve but never \\
& & normalizes \\
Clinical symptoms & Intermittent and variable & Persistent \\
\hline
\end{tabular}

In 2005, 22\% of Canadians aged 12 years and older still smoked. Although approximately $41 \%$ of smokers try to quit smoking each year, only about $10 \%$ achieve and maintain abstinence. At least $70 \%$ of smokers visit a physician each year and advice is an important motivator to quit. Smoking cessation reduces the rate of lung function decline, confers a survival advantage and leads to symptomatic improvement in COPD patients. Minimal interventions, lasting less than $3 \mathrm{~min}$, should systematically be offered to every smoker; however, more intensive counselling with pharmacotherapy results in higher quit rates and should be used whenever possible. The use of medication, including nicotine replacement therapy and the antidepressant bupropion, approximately doubles 
Oxygen

Inhaled corticosteroids / LABA

Pulmonary rehabilitation

Long-acting bronchodilator(s)

PRN short-acting bronchodilator(s)

Smoking cessation/exercise/self-management/education

Lung function impairment scale

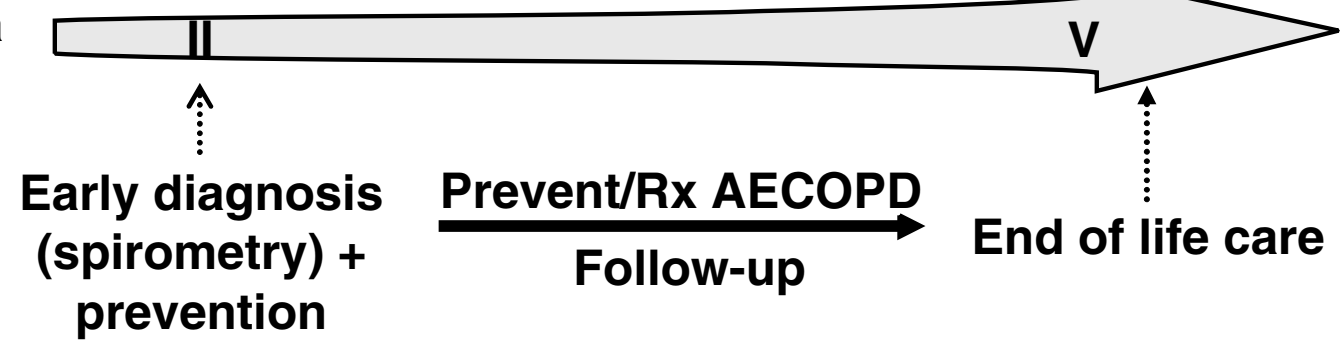

Figure 1) A comprehensive approach to the management of chronic obstructive pulmonary disease (COPD). AECOPD Acute exacerbation of COPD; LABA Long-acting beta ${ }_{2}$-agonist; MRC Medical Research Council; PRN As needed; Rx Treatment

smoking cessation rates. A new nicotinic acetylcholine partial agonist, varenicline, has superior efficacy to bupropion and placebo.

Vaccinations

Vaccinations prevent acute exacerbations of COPD (AECOPD).

Individuals with COPD infected with influenza are at significant risk of requiring hospitalization. Annual influenza vaccination reduces morbidity and mortality from the disease by as much as $50 \%$ in elderly patients, and reduces the incidence of hospitalization by as much as $39 \%$ in patients with chronic lung disease. The benefit of pneumococcal vaccine in COPD is less well-established but the Panel recommends that it be given at least once and possibly repeated in five to 10 years.

\section{Pharmacotherapy}

Bronchodilators form the mainstay of pharmacological therapy for COPD. They decrease airway smooth muscle tone, thus improving expiratory flow rates and reducing hyperinflation, resulting in reduced dyspnea, improved exercise tolerance and health status. Optimal pharmacotherapy of COPD is guided on an individual basis by assessment of level of disease severity (ie, symptoms/disability and spirometry) and frequency of acute exacerbations (Figure 2).

- Short-acting bronchodilators, both anticholinergics and beta $_{2}$-agonists, have been shown to improve pulmonary function, dyspnea and exercise performance in patients with moderate to severe COPD. They have inconsistent impact on health status. Individual responses to the different classes of bronchodilators are variable.

- Long-acting beta ${ }_{2}$-agonists (LABAs) (eg, salmeterol $50 \mu \mathrm{g}$ twice daily or formoterol $12 \mu \mathrm{g}$ twice daily) offer more sustained improvements in pulmonary function, chronic dyspnea and health status than short-acting bronchodilators in patients with moderate to severe COPD. However, the effects of LABAs on exercise performance have been inconsistent. In the Towards a Revolution in COPD Health (TORCH) study, salmeterol alone was not associated with reduced mortality, but was associated with reduced frequency and severity of exacerbations compared with placebo. Inhaled beta $2_{2}$-agonists are generally well-tolerated. The most common adverse effects include tachycardia, 


\section{Increasing Disability and Lung Function Impairment}

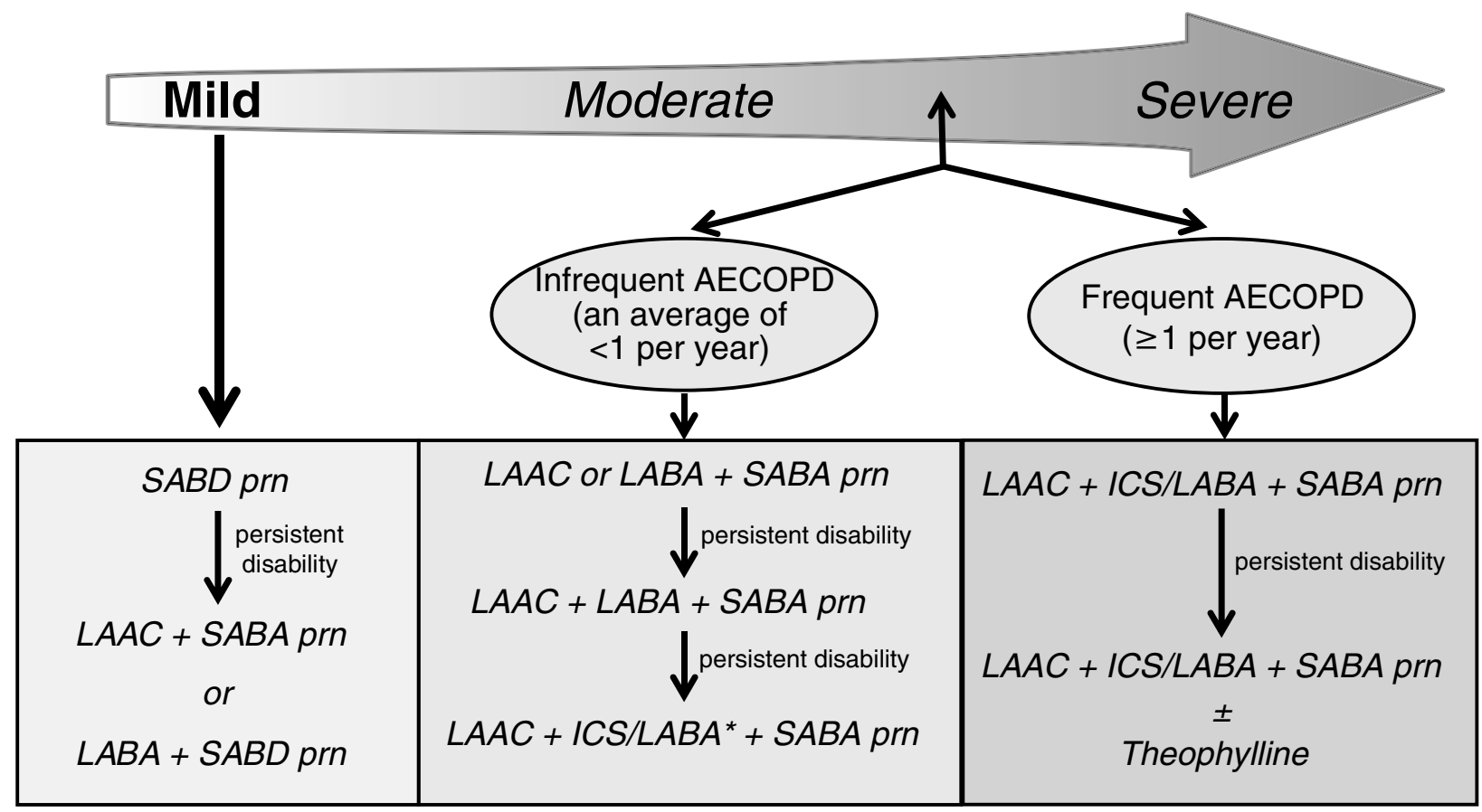

Figure 2) Recommendations for optimal pharmacotherapy in chronic obstructive pulmonary disease (COPD). *Refers to the lower dose inhaled corticosteroid/long-acting beta 2 -agonist (ICS/LABA). AECOPD Acute exacerbation of COPD; LAAC Long-acting anticholinergic; prn As needed;

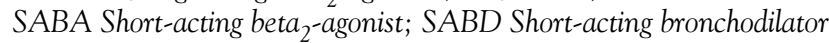

palpitation, irritability, insomnia, muscle cramps and tremor. More serious side effects are rare.

- Long-acting anticholinergics (LAACs) (eg, tiotropium bromide $18 \mu \mathrm{g}$ once daily) have more sustained effects on pulmonary function, chronic activity-related dyspnea and health status compared with regular dose ipratropium bromide or placebo in patients with moderate to severe COPD. Tiotropium provides improvements in lung hyperinflation, exercise endurance, exacerbations and health resource utilization when compared with placebo in patients with moderate to severe COPD. When compared with LABAs, tiotropium provides greater

improvements in lung function and is associated with larger improvements in dyspnea and health status. Apart from occasional dry mouth, inhaled anticholinergic drugs are generally well tolerated. Rarely, prostatic symptoms, glaucoma (if the drug gets directly into the eye) and supraventricular arrythmias have been reported, necessitating drug discontinuation.

- Combined long-acting bronchodilators: Two small, short-term studies have provided evidence that the combination of LAAC and LABA bronchodilators may have additive sustained effects on pulmonary function in patients with moderate to severe COPD. In a recent one-year Canadian study, the addition of salmeterol to tiotropium in patients with more advanced COPD was associated with consistent improvement in health status but not with significant improvement in spirometry or reduction in the frequency and severity of exacerbation compared with tiotropium alone.

- Inhaled corticosteroids (ICS) alone: Unlike asthma, the role for ICS (as monotherapy) in COPD is controversial. ICS do not have consistent effects on indices of airway inflammation, pulmonary function, symptoms, frequency or severity of exacerbation, and health status in COPD. ICS alone is generally inferior to an ICS/LABA combination for all of the above outcomes. Randomized clinical trials have not shown any benefit on the relentless decline in lung function that is characteristic of COPD. Adverse effects of ICS include dysphonia, oral candidiasis and skin bruising. Long-term use of high-dose ICS may be associated with a reduction in bone density, posterior subcapsular cataracts, glaucoma and pneumonia.

- ICS/LABA combinations: Two combination ICS and LABA products are currently available in 
TABLE 4

Potential preventive strategies for acute exacerbations of chronic obstructive pulmonary disease (AECOPD)

\begin{tabular}{l}
\hline Strategies \\
\hline - Smoking cessation \\
Vaccinations \\
oInfluenza (annually) \\
oPneumococcal vaccine (every five to 10 years) \\
- Self-management education \\
- Regular long-acting bronchodilator therapy (moderate to severe \\
COPD) \\
- Regular therapy with ICS/LABA combination (moderate to severe COPD \\
- Oral corticosteroid therapy for AECOPD \\
- Pulmonary rehabilitation \\
\hline
\end{tabular}

ICS Inhaled corticosteroids; LABA Long-acting beta ${ }_{2}$-agonist

Canada: fluticasone plus salmeterol (Advair, GlaxoSmithKline Inc, Canada), and budesonide plus formoterol (Symbicort, AstraZeneca Canada Inc). Studies have confirmed that lower dose ICS/LABA (ie, Advair 250/50 $\mu$ g twice daily) have consistent beneficial effects on pulmonary function and exercise endurance compared with placebo. The TORCH study compared combined ICS/LABA (Advair 500/50 $\mu$ g twice daily) with placebo, and ICS or LABA used alone. The probability of all-cause mortality at three years was $15.2 \%$ in the placebo group and $12.6 \%$ in the ICS/LABA group (17\% relative reduction or $2.6 \%$ absolute risk reduction) $(\mathrm{P}=0.052)$. This study demonstrated that treatment with ICS/LABA improved secondary outcome measures (exacerbation reduction, improvement of pulmonary function and health status) in comparison with placebo, LABA and ICS alone. The Canadian, multicentre, placebo-controlled trial studied the effectiveness of adding LABA (ie, salmeterol) or ICS/LABA (ie, Advair) to LAAC (ie, tiotropium) in patients with moderate to severe COPD for one year. Adding ICS/LABA to tiotropium did not significantly improve overall exacerbation rates; however, benefits were demonstrated on secondary outcomes (lung function, quality of life and hospitalization rates).

- Oral theophyllines are relatively weak bronchodilators that offer modest improvements in pulmonary function, dyspnea and exercise performance. The addition of oral theophyllines to inhaled bronchodilator therapy may offer benefits in some patients, although the evidence is very limited. Benefits of theophyllines need to be weighed against the risk of serious cardiovascular and neurological side effects. Drug interactions are common and it is necessary to monitor drug levels with blood tests.
- Oral corticosteroids: Approximately 10\% of patients with stable COPD will demonstrate a 20\% improvement in $\mathrm{FEV}_{1}$ with oral corticosteroids. This modest benefit must be weighed against the risk of numerous, serious adverse effects associated with maintenance use of systemic corticosteroids, including adrenal suppression, osteoporosis, cataract formation, skin thinning, muscle weakness, hypertension, diabetes and psychosis. Long-term use of low-dose oral steroids is not recommended in COPD.

\section{ACUTE EXACERBATIONS}

AECOPD are associated with high costs of care, increased health resource utilization, accelerated decline in lung function, poorer health-related quality of life and increased mortality. An AECOPD is defined as a sustained worsening of dyspnea, cough or sputum production leading to an increase in the use of maintenance medications and/or supplementation with additional medication. Management and prevention of AECOPD is critically important in the optimal care of COPD.

At least one-half of AECOPD are thought to be infectious in nature. Other triggering factors include congestive heart failure, exposure to allergens and irritants, and pulmonary embolism. The average COPD patient experiences approximately two exacerbations per year but this rate is highly variable among individuals. Potential strategies to prevent AECOPD are presented in Table 4.

Management of AECOPD requires careful history, physical examination and limited laboratory investigations (ie, chest radiograph in those presenting to emergency department; arterial blood gases in patients with low oxygen levels on oximetry). Combined, increased doses of inhaled short-acting beta $_{2}$-agonist (SABA) and an anticholinergic should be used to improve pulmonary function and dyspnea. Oral or parenteral corticosteroids (dosages of $25 \mathrm{mg}$ to $50 \mathrm{mg}$ of prednisone equivalent per day for between seven and 14 days) are recommended in most patients with moderate to severe AECOPD. Antibiotics are beneficial to treat more severe purulent AECOPD (new increased expectoration of mucopurulent sputum and dyspnea). In choosing antibiotics, patients can be divided into simple or complicated exacerbations, based on the presence of risk factors that increase the likelihood of treatment failure or are more likely to be associated with virulent or resistant microbial pathogens (Table 5).

\section{PULMONARY REHABILITATION}

All patients with COPD should be encouraged to maintain an active lifestyle. Clinically stable patients who remain dyspneic and limited in their exercise capacity despite optimal pharmacotherapy should be referred for supervised pulmonary rehabilitation. Pulmonary rehabilitation significantly improves dyspnea, exercise endurance, quality of life and risk of hospitalizations following AECOPD. Where formal rehabilitation programs are not available, patients should be encouraged to undertake a home-based exercise program to prevent progressive skeletal muscle deconditioning associated with inactivity. 
TABLE 5

Antibiotic treatment recommendations for purulent acute exacerbations of chronic obstructive pulmonary disease (COPD)

\begin{tabular}{|c|c|c|c|c|}
\hline Group & Basic clinical state & Symptoms and risk factors & Probable pathogens & First choice \\
\hline Simple exacerbation & COPD without risk factors & $\begin{array}{l}\text { Increased sputum purulence } \\
\text { and dyspnea }\end{array}$ & $\begin{array}{l}\text { Haemophilus influenzae, } \\
\text { Haemophilus species, } \\
\text { Moraxella catarrhalis, } \\
\text { Streptococcus pneumoniae }\end{array}$ & $\begin{array}{l}\text { Amoxicillin, second- or third- } \\
\text { generation cephalosporins, } \\
\text { doxycycline, extended- } \\
\text { spectrum macrolides, } \\
\text { trimethoprim/sulfamethoxazole } \\
\text { (in alphabetical order) }\end{array}$ \\
\hline Complicated exacerbation & COPD with risk factors & $\begin{array}{l}\text { As in simple plus at least one of: } \\
\text { - } \mathrm{FEV}_{1}<50 \% \text { predicted } \\
\text { - } \geq 4 \text { exacerbations per year } \\
\text { - Ischemic heart disease } \\
\text { - Use of home oxygen } \\
\text { - Chronic oral steroid use }\end{array}$ & $\begin{array}{l}\text { As in simple plus: } \\
\text { Klebsiella species and } \\
\text { other Gram-negatives } \\
\text { Increased probability of } \\
\text { beta-lactam resistance } \\
\text { Pseudomonas species }\end{array}$ & $\begin{array}{l}\text { Fluoroquinolone, beta-lactam/ } \\
\text { beta-lactamase inhibitor } \\
\text { (in order of preference) }\end{array}$ \\
\hline
\end{tabular}

Repeat prescriptions of the same antibiotic class should be avoided within a three-month interval. FEV 1 Forced expiratory volume in $1 \mathrm{~s}$

\section{OXYGEN THERAPY}

Long-term continuous oxygen (15 h/day or more to achieve an oxygen saturation of $90 \%$ or greater) offers a survival advantage to patients with stable COPD with severe hypoxemia (partial pressure of arterial oxygen $55 \mathrm{mmHg}$ or less), or when partial pressure of arterial oxygen is less than $60 \mathrm{mmHg}$ in the presence of bilateral ankle edema, cor pulmonale or a hematocrit of greater than $56 \%$.

\section{SPECIALIST REFERRAL}

Family physicians have a primary role in the management of COPD; however, referral to a specialist is appropriate in certain circumstances (Table 6).

Specialists can also assist in the management of patients who fail to respond to bronchodilator therapy, and those who require pulmonary rehabilitation, an assessment for oxygen therapy, or surgical intervention (ie, bullectomy, lung volume reduction surgery, lung transplantation).

\section{END-OF-LIFE ISSUES}

COPD is a progressive, disabling condition that may ultimately result in respiratory failure and death. Physicians have a responsibility to help patients with COPD and their cargivers to plan for the end of life and to make sure the necessary support is in place to assist them through this critical terminal phase.

Physicians need to improve their understanding of the issues facing patients and their informal caregivers through honest, informative, empathetic conversations that can form the basis for effective advanced care planning. Features of advanced disease that increase the likelihood of death from an episode of acute exacerbation include very severe airflow obstruction (ie, $\mathrm{FEV}_{1}$ less than $30 \%$ predicted; inspiratory capacity less than $80 \%$ predicted), poor functional status (ie, Medical Research Council dyspnea scale [MRC] grades 4 to 5), poor nutritional status (ie, body mass index less than $19 \mathrm{~kg} / \mathrm{m}^{2}$ ), presence of pulmonary hypertension and recurrent severe acute exacerbations
TABLE 6

When to refer to a specialist

Diagnostic uncertainty
Symptoms are disproportionate to level of airflow obstruction
Accelerated decline of pulmonary function
Suspicion of alpha ${ }_{1}$-antitrypsin deficiency
Onset of symptoms at a young age
Severe or recurrent acute exacerbations of chronic obstructive pulmonary
disease
Failure to respond to therapy

requiring hospitalization. These patients in particular will benefit from timely discussions about end-of-life issues. Discussions that underscore the value of palliative services and the effective alleviation of terminal dyspnea help to reduce anticipatory anxiety.

\section{Case 1}

\section{ILLUSTRATIVE CASE SCENARIOS}

A 55-year-old woman comes to clinic for her annual checkup. She has been getting recurrent 'colds' over the past year. She notes shortness of breath on climbing hills over the past year. She has a long history of smoking.

Assessment: This patient requires a thorough clinical assessment, including spirometry to confirm the suspicion that she has COPD. Spirometry demonstrates an $\mathrm{FEV}_{1}$ of $80 \%$ predicted and an $\mathrm{FEV}_{1} /$ forced vital capacity of 0.67 , indicating mild airflow obstruction. She has MRC grade 2. This patient has mild COPD.

Suggested therapy (Table 7, Figures 1 and 2): Treatment should consist of educating the patient about her illness, providing smoking cessation counselling/pharmacotherapy, administering vaccinations and encouraging regular physical activity. In terms of pharmacotherapy, initiation of as needed, inhaled, short-acting bronchodilator therapy is recommended. Options include SABA (which is of rapid onset and can be used prophylactically in anticipation of exercise), short-acting anticholinergics, alone or in 


\section{TABLE 7 \\ Management of symptomatic milder chronic obstructive pulmonary disease ${ }^{*}$}

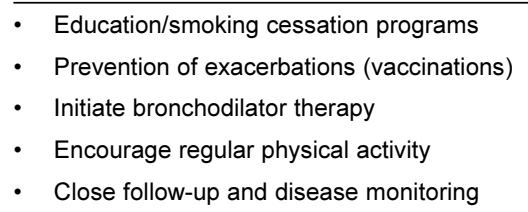

*Medical Research Council grade 2 and/or forced expiratory volume in $1 \mathrm{~s}$ $\left(F E V_{1}\right) /$ forced vital capacity $<0.70, F E V_{1} \geq 80 \%$ predicted

combination. The choice of first-line therapy in mild symptomatic COPD should be individualized, based on clinical response and tolerance of side effects. If dyspnea and exercise intolerance persist, a long-acting bronchodilator of either class (LAAC or LABA) could be intiated with use of a short-acting bronchodilator as needed.

\section{Case 2}

You are seeing a 67-year-old man in clinic who is an exsmoker with well-documented COPD. His $\mathrm{FEV}_{1}$ is $55 \%$ predicted. Medications include ipratropium bromide and salbutamol inhalers, each taken four times daily. Despite this, he remains short of breath walking $50 \mathrm{~m}$ to $75 \mathrm{~m}$ on level ground at a slow pace. As a result, he has given up golf and playing with his grandchildren. He has not suffered an exacerbation in four years.

Assessment: This patient has an MRC grade 4 and moderate airflow obstruction indicative of moderate severity $\mathrm{COPD}$, which is having a negative impact on his activity level and quality of life.

Suggested therapy (Figures 1 and 2): Treatment should consist of educating the patient about his illness, administering vaccinations and encouraging regular physical activity. In terms of pharmacotherapy, a long-acting bronchodilator should be initiated - either a LAAC (ie, tiotropium bromide $18 \mu \mathrm{g}$ once daily) instead of ipratropium bromide or a LABA (ie, formoterol $12 \mu \mathrm{g}$ twice daily or salmeterol $50 \mu$ twice daily) to improve dyspnea, exercise endurance and health status. SABAs may be used as needed for immediate symptom relief. A combination of tiotropium and a LABA is recommended if dyspnea and exercise intolerance persist. If symptoms still persist, consideration for replacing the LABA with a lower dose ICS/LABA combination is warranted (ie, Advair 50/250 $\mu \mathrm{g}$ twice daily or Symbicort 12/200 $\mu$ g twice daily). If the patient remains symptomatic despite optimal pharmacotherapy, then referral for pulmonary rehabilitation is appropriate.

\section{Case 3}

A 71-year-old woman, who is an ex-smoker with welldocumented COPD, presents to the emergency department with three days increased dyspnea, cough and purulent sputum. She has experienced similar episodes on two previous occasions over the past three years. At baseline, she is dyspneic when combing her hair or getting dressed. Her FEV is $35 \%$ predicted. Medications include tiotropium bromide $18 \mu \mathrm{g}$ once daily and as needed salbutamol.
Assessment: This patient has MRC grade 5 and severe airflow obstruction indicative of severe COPD, which is having a negative impact on her activity level and quality of life. She is experiencing frequent acute exacerbations. Clinical assessment in the emergency department confirms that another AECOPD explains the current presentation. Arterial blood gases show no evidence of hypercapnia but an oxygen saturation of $85 \%$ on room air.

Suggested therapy (Table 5): Acutely, this patient will require admission to hospital for controlled oxygen therapy (to maintain oxygen saturation at $88 \%$ to $92 \%$ ); combined, inhaled SABA and anticholinergic; systemic corticosteroids (eg, prednisone $25 \mathrm{mg}$ to $50 \mathrm{mg}$ orally daily for seven to 14 days); and an antibiotic, either a respiratory fluoroquinolone or beta-lactam/beta-lactamase inhibitor (eg, amoxicillin/clavulanic acid). Long-term treatment should consist of educating the patient about her illness, administering vaccinations and referral for pulmonary rehabilitation (Figures 1 and 2). In terms of pharmacotherapy, a LAAC (ie, tiotropium bromide $18 \mu \mathrm{g}$ once daily) and higher-dose ICS/LABA combination is warranted

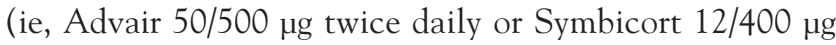
twice daily) to improve dyspnea, exercise endurance, health status and reduce exacerbation frequency. SABAs may be used as needed for immediate symptom relief. If symptoms persist, a long-acting preparation of oral theophylline may be tried, although monitoring of blood levels, side effects and potential drug interactions is necessary. This patient would benefit from discussion about end-of-life issues.

Please refer to the Canadian Respiratory Journal, Volume 14, Supplement B for the complete 2007 update, including references [Canadian Thoracic Society recommendations for management of chronic obstructive pulmonary disease - 2007 update. Can Respir J 2007;14(Suppl B):5B-32B]. For more information on the Canadian Thoracic Society COPD Guidelines, or to request implementation tools for clinicians and educators, please, visit our Web site at www.COPDguidelines.ca.

SPONSORING ORGANIZATIONS: The Canadian College of Family Physicians, The Family Physicians Airway Group of Canada, The Lung Association, Canadian Nurses' Respiratory Society, Canadian Physiotherapy Cardio-Respiratory Society, Respiratory Therapy Society of The Lung Association and the Canadian COPD Alliance.

COMPETING INTERESTS: Collectively, the physicians on the Scientific Review Panel have on at least one occasion acted as consultants for, received research funds from, received speaker's fees from, and received travel assistance from the various pharmaceutical companies listed below.

FUNDING: These guidelines were developed under the auspices of the Scientific Review Panel of the Canadian Thoracic Society. This process was facilitated by funding from ALTANA Pharma Inc, AstraZeneca Canada Inc, Bayer Canada Inc, Boehringer Ingelheim (Canada) Inc, Bristol-Myers Squibb, GlaxoSmithKline Inc and Pfizer. None of the pharmaceutical sponsors played a role in the analysis or interpretation of the scientific data or in any decision regarding recommendations. 


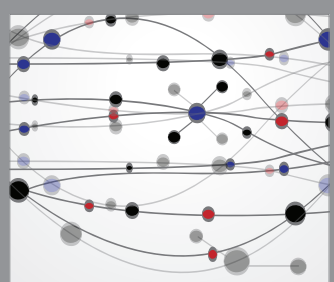

The Scientific World Journal
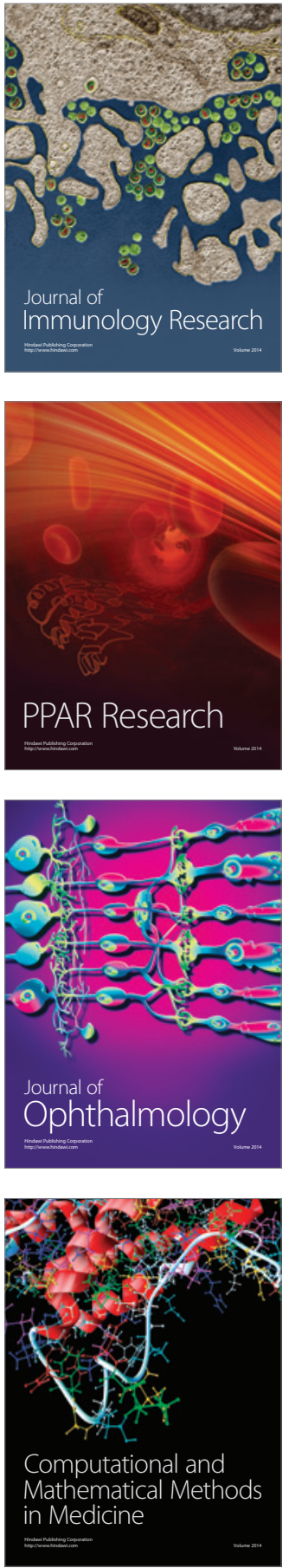

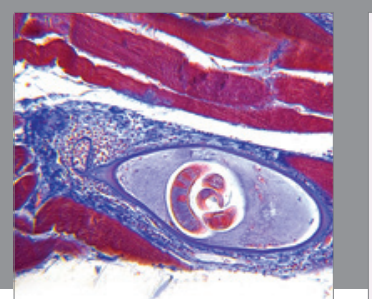

Gastroenterology Research and Practice

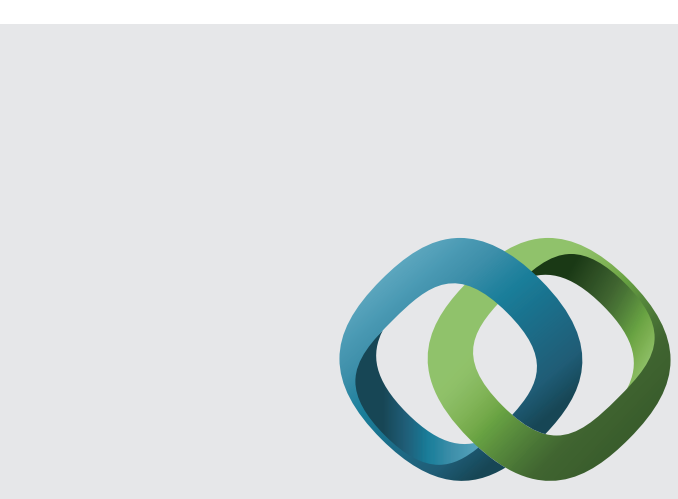

\section{Hindawi}

Submit your manuscripts at

http://www.hindawi.com
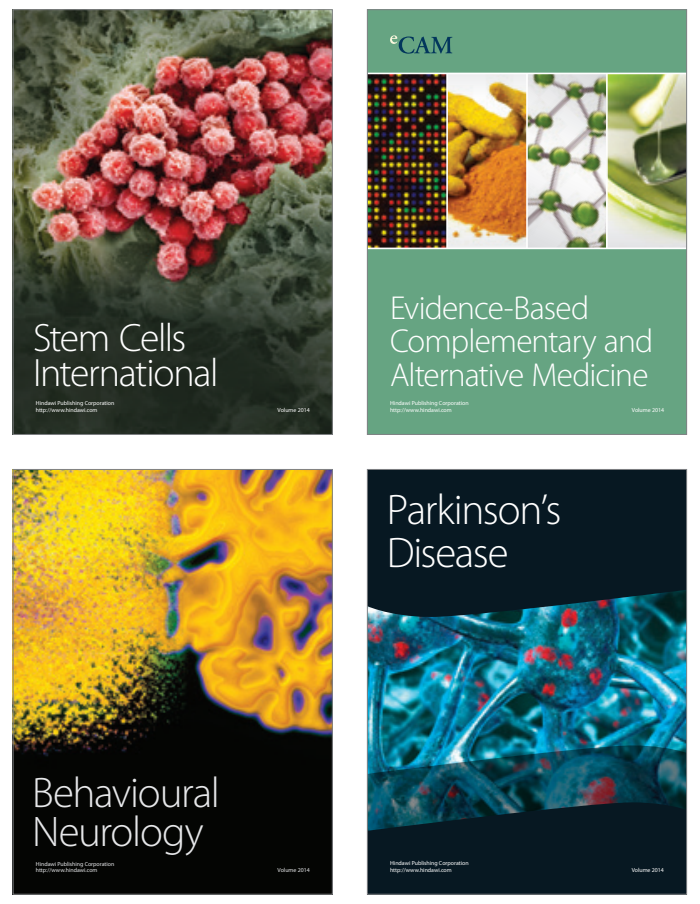
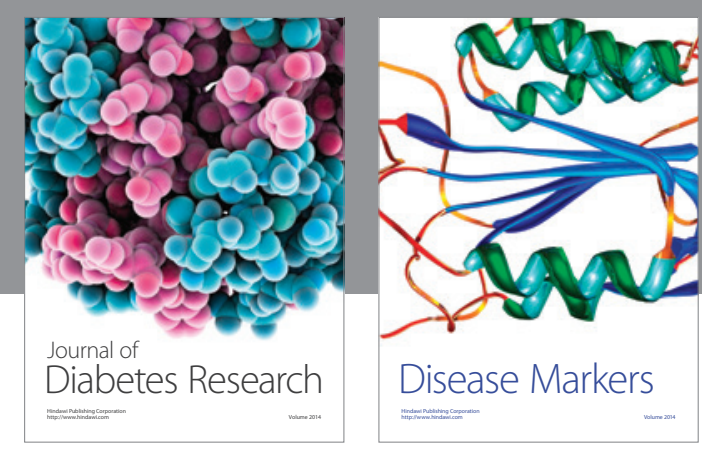

Disease Markers
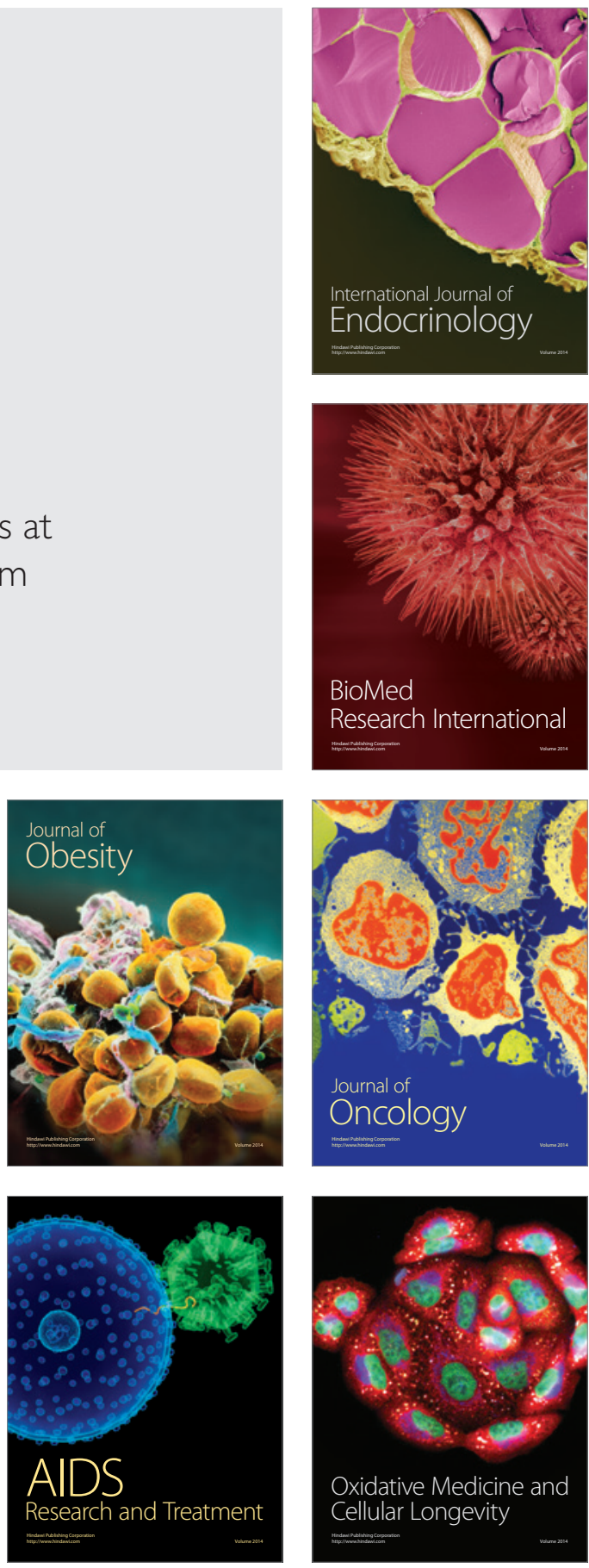